\title{
Femtosecond dopant-to-solvent energy transfer inside helium nanodroplets
}

\author{
Bernhard Thaler ${ }^{1}$, Miriam Meyer ${ }^{1}$, Pascal Heim ${ }^{1}$, Leonhard Treiber ${ }^{1}$, Wolfgang E. Ernst ${ }^{1}$, \\ and Markus Koch $^{1, *}$ \\ ${ }^{1}$ Graz University of Technology, Institute of Experimental Physics, 8010 Graz, Austria
}

\begin{abstract}
Photoexcitation of atoms solvated inside superfluid helium nanodroplets is investigated with femtosecond photoelectron spectroscopy. Expansion of the solvation shell within about $600 \mathrm{fs}$ is observed as transient photoelectron peak shift. For larger photoexcitation energies within the blueshifted absorption band, more energy is transferred to the He droplet and the expansion proceeds faster.
\end{abstract}

\section{Introduction}

Superfluid helium nanodroplets $\left(\mathrm{He}_{\mathrm{N}}\right)$ are widely used to create and investigate tailor-made or fragile molecular systems at $0.4 \mathrm{~K}$ temperature. Most dopants are solvated inside the droplet where they reside in a He bubble that forms because of strong Pauli repulsion between the dopant electrons and the surrounding He. Electronic excitation spectra of dopants consist of broadened and blue-shifted bands, associated to expansion of the valence electron wave function and the corresponding bubble enlargement. Recently, we investigated the photoexcitation dynamics of indium (In) atoms inside $\mathrm{He}_{\mathrm{N}}$ with femtosecond time resolution and identified the following three processes [1]: expansion of the He bubble below $1 \mathrm{ps,}$ bubble oscillation with a period of $\sim 30 \mathrm{ps}$, and ejection of the In atom from the droplet after $\sim 60 \mathrm{ps}$.

Here, we investigate the dependence of the bubble expansion on the photoexcitation energy by monitoring the corresponding transfer of excess photoexcitation energy from the dopant to the solvent.

\section{Method}

As described in detail previously [1,2], He $\mathrm{N}_{\mathrm{N}}$ with an average size of $\bar{N}=4800$ atoms are generated in a supersonic expansion and doped with single In atoms by passing a resistively heated pickup cell. Dynamics of the In-He system are investigated with a femtosecond pump-probe photoionization experiment with variable time-delay, where the kinetic energy of photoelectrons (PEs) is measured with a time-of-flight magnetic bottle type spectrometer. Pump pulses are obtained from a commercial Ti:sapphire laser system and frequency upconverted by an optical parametric amplifier to photon energies in the range of 3.0-3.6 eV

\footnotetext{
* Corresponding author: markus.koch@tugraz.at
} 
(413-344 nm) in order to excite the In $6{ }^{2} S_{1 / 2} \leftarrow 5{ }^{2} P_{1 / 2}$ band inside $\mathrm{He}_{\mathrm{N}}$ [2]. Probe pulses are frequency doubled to $3.046 \mathrm{eV}(407 \mathrm{~nm})$.

\section{Results and Discussion}

Figure 1a shows the In-He $\mathrm{N}_{\mathrm{N}}$ excitation spectrum in the range of the In $6{ }^{2} S_{1 / 2} \leftarrow 5{ }^{2} P_{1 / 2}$ transition, which consists of two bands. The stronger band centred at $3.36 \mathrm{eV}$, which is blueshifted by $\sim 335 \mathrm{meV}$ relative to the bare-atom transition (black line), is allocated to the excitation of fully solvated In atoms [2]. The weak band at $\sim 3.12 \mathrm{eV}$ corresponds to excitation of surface-located atoms, and the signal increase above $3.5 \mathrm{eV}$ results from an In dimer band [2], both of which are not of interest here. The large shift of the in-droplet band indicates an exceptionally pronounced interaction with the He solvent upon photoexcitation of the In atom, compared to other metal atoms (e.g., Ref. [5]).
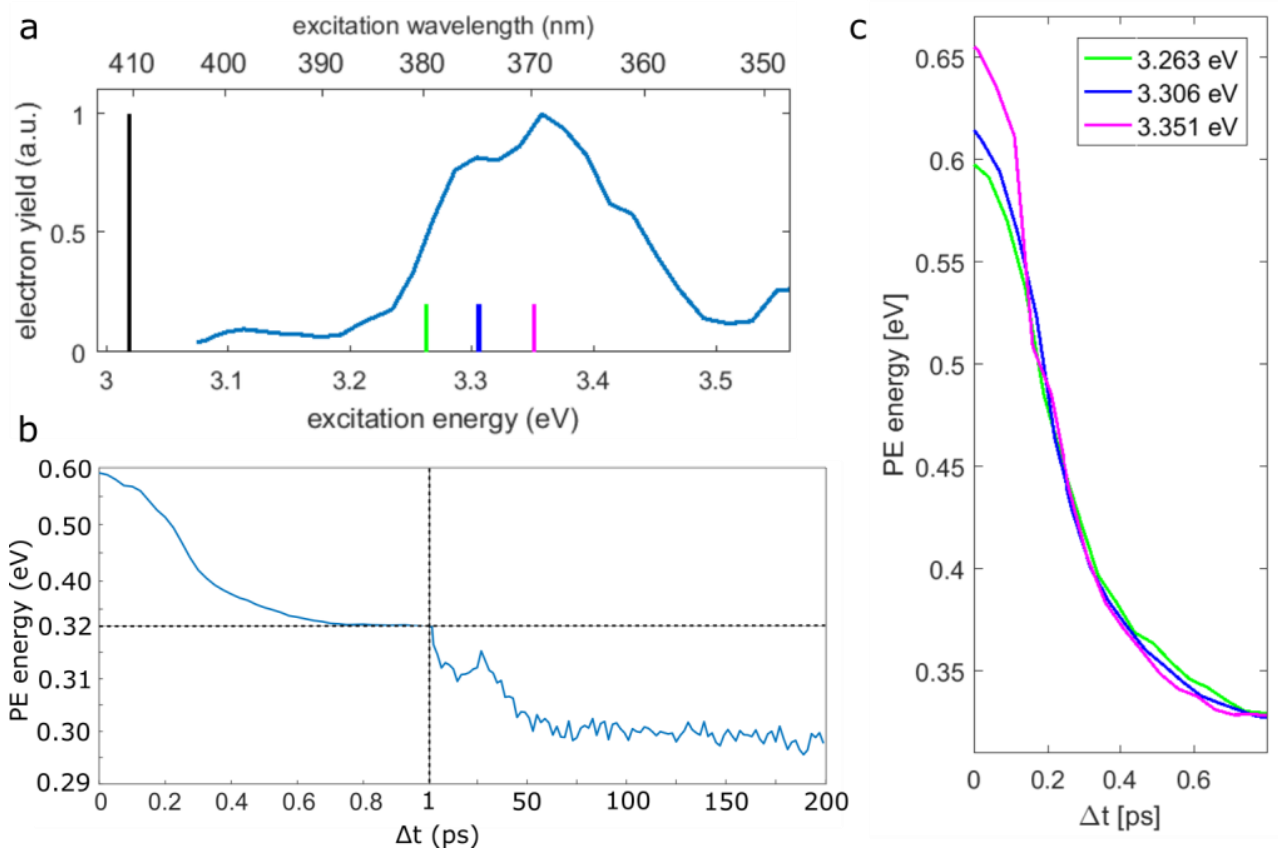

Figure 1: Photoexcitation of the In-HeN system in the range of the In $6{ }^{2} S_{1 / 2} \leftarrow 5{ }^{2} P_{1 / 2}$ transition. (a) Excitation spectrum recorded at a fixed pump-probe time delay of $200 \mathrm{ps}$ by scanning the pump photon energy and monitoring the monomer peak of the PE spectrum [2]. The black line represents the bareatom transition. (b) Transient $6{ }^{2} S_{1 / 2}$ PE peak position for $3.297 \mathrm{eV}$ excitation energy, determined by fitting Gaussian functions to the PE spectra. (Note that the first picosecond is displayed with higher time resolution and lower energy resolution. (c) Transient PE peak position within the first 800 fs for different photoexcitation energies, as indicated in (a).

The excited state ionization energy of the dopant shows a strong dependence on the configuration of the He environment around the dopant, making the PE peak position a sensitive probe to follow the time evolution of the solvation shell rearrangement [1]. The transient PE peak position corresponding to ionization of the In $6{ }^{2} S_{1 / 2}$ state (Figure 1b), shows an initially fast and monotonic decrease of $280 \mathrm{meV}$ to an absolute value of $0.32 \mathrm{eV}$ at $1 \mathrm{ps}$. This is followed by a slower decrease of $20 \mathrm{meV}$ to the bare-atom PE energy of $300 \mathrm{meV}$ [3] between $1 \mathrm{ps}$ and $\sim 60 \mathrm{ps}$, together with a temporary increase around $\sim 30 \mathrm{ps}$. 
The fast initial decrease represents the bubble expansion and is caused by a timedependent increase of the ionization energy. The latter results from an energetic decrease of the In-He $\mathrm{H}_{\mathrm{N}}$ excited state and an increase of the ionic state over time [1]. After the He bubble has adapted to the excited state electron wave function of the In atom, at around $1 \mathrm{ps}$, the PE energy is still $20 \mathrm{meV}$ above the bare-atom value, representing the decreased ionization energy in the He environment due to polarization effects [4]. Consequently, the remaining decrease of the PE energy between 1 ps and $\sim 60$ ps reflects the ejection process of the In atom in its repulsive excited state from the droplet, under consideration of the ensemble distribution of In locations inside the droplets. Photoexcitation also stimulates an oscillation of the He bubble and its first contraction is observed as temporal increase of the PE peak position at $\sim 30$ ps caused by the temporary increase of He density in the vicinity of the In atom.

Figure 1c shows PE peak transients obtained with different photoexcitation energies, as indicated by markers of the same color in Figure 1a. The expected trend of higher excitation energies leading to larger PE energies is confirmed: For applied photon energies of 3.263, 3.306 and $3.351 \mathrm{eV}$ time-zero peak positions of about 598, 615 and $656 \mathrm{meV}$ are observed. These values are slightly below the expected values of 610, 653 and $698 \mathrm{meV}$ (calculated from the sum of pump and probe photon energies minus the In ionization energy of $5.786 \mathrm{eV}$ [3], minus the reduction of ionization potential inside the $\mathrm{He}_{\mathrm{N}}$ of $\sim 87 \mathrm{meV}$ [1]), which might be caused by an increased ionization rate within the pump-probe cross-correlation.

While all transients in Figure 1c level off at the same PE energy of $320 \mathrm{meV}$ after $1 \mathrm{ps}$, the transients starting at a higher PE energy show a faster decrease. For the same photon energy sequence as before, $90 \%$ of the shift is completed after about 500, 550 and $580 \mathrm{fs}$, respectively. An increase of the photoexcitation energy by $90 \mathrm{meV}$ can be estimated to shorten the bubble expansion by $\sim 80 \mathrm{fs}$, corresponding to $15 \%$. In a simple picture considering potential energy surfaces as function of the bubble radius, this indicates that the excited state wave packet is produced at higher potential energies for increased photon energies, resulting in faster nuclear dynamics.

In conclusion, we have shown that the photoexcitation dynamics of dopants inside $\mathrm{He}_{\mathrm{N}}$ can be well resolved with femtosecond photoelectron spectroscopy, and that the amount of excess energy during photoexcitation determines the kinetics of the solvation shell dynamics.

We acknowledge financial support by the Austrian Science Fund (FWF) under Grant P29369-N36, as well as support from NAWI Graz.

\section{References}

1. B. Thaler et al., accepted by Nat. Comm., arXiv preprint arXiv:1804.04497 (2018)

2. B. Thaler, R. Meyer, P. Heim, S. Ranftl, J.V. Pototschnig, A.W. Hauser, M. Koch, W.E. Ernst, submitted (2018)

3. A. Kramida, Yu. Ralchenko, J. Reader and and NIST ASD Team. NIST Atomic Spectra Database, https://physics.nist.gov/asd (2018)

4. E. Loginov, D. Rossi, M. Drabbels, PRL 95, 163401 (2005)

5. A. Kautsch, M. Hasewend, M. Koch, W. E. Ernst, PRA 86, 33428 (2012) 